NuMI-L-523

$8 / 24 / 99$

\title{
MINOS Near-Detector Coil Design
}

\author{
J. K. Nelson" ${ }^{\mathrm{a}}$ J. Kilmer ${ }^{\mathrm{b}}$ \\ aDept. of Physics, College of William \& Mary, Williamsburg, VA 23187, USA \\ ${ }^{\mathrm{b}}$ Fermi National Accelerator Laboratory, Batavia, IL 60510, USA
}

\section{Introduction}

This document has been prepared for the 8/99 Conceptual Design Review of the MINOS near detector coil. It's main goal is to provide a set of references to previous documents and to assemble various design drawings and engineering calculations that have not been included in previous technical memos. It also provides some background material relevant for the coil implementation. Much of the text for this document is edited from the MINOS Detectors Technical Design Report [1].

\section{Overview of the Near Detector}

The 980-ton MINOS near detector will be installed in the new NuMI near hall at 100m below grade at Fermilab. It will be assembled from 282 1-inch thick steel plates. The planes are made from low carbon (1006), hot rolled steel and are toroidally magnetized. Each plane will be hung by two "ears," which are extensions of the octagonal plane structure, similar to the hanging files in a file drawer. The plates have a center-to-center spacing of $5.94 \mathrm{~cm}$.

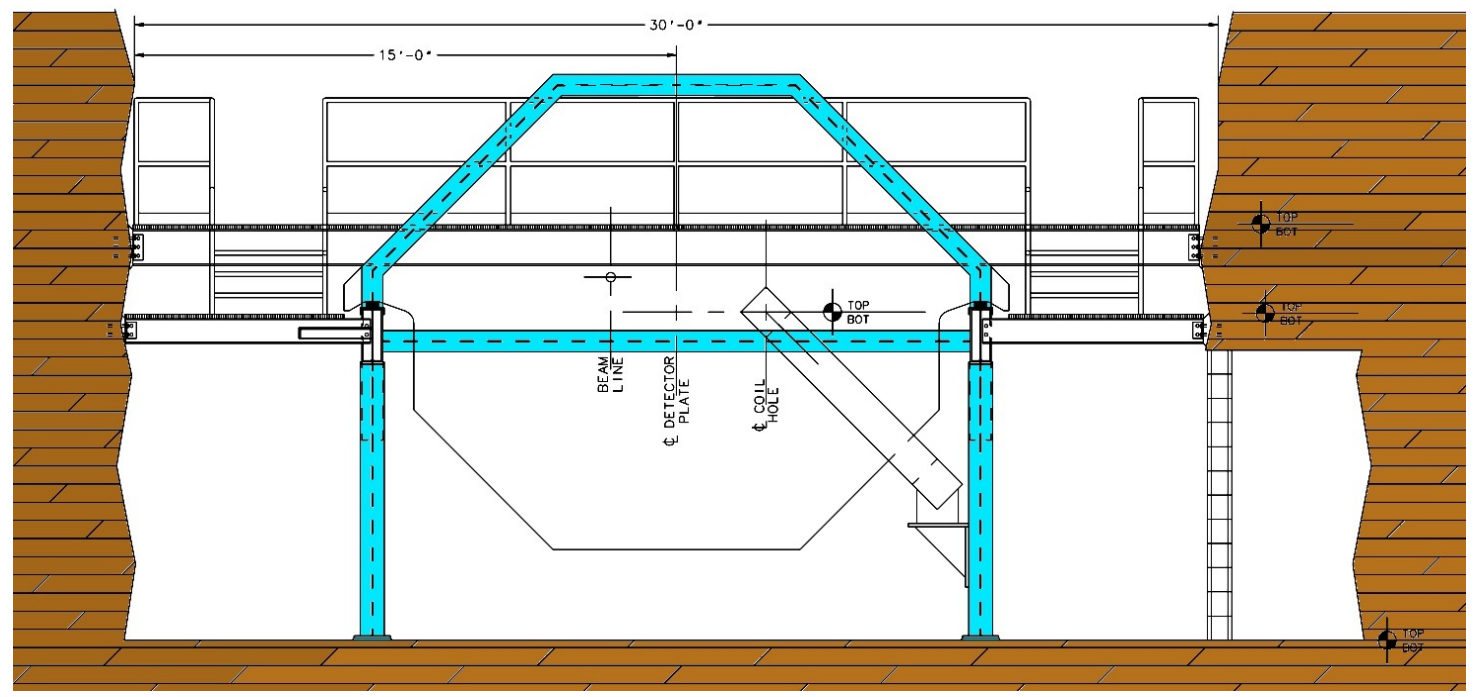

Figure 1: Sketch of a MINOS near detector steel plane hanging from the detector support rails. Also shown are the detector support structure, the side walkways, and the magnet coil. 
A hole is provided in each plate for the magnet coil. It is offset about $0.6 \mathrm{~m}$ from the center of the plane. Because the neutrino beam at the near detector will only be about $1 \mathrm{~m}$ in diameter, the detector plane geometry is designed to reduce the area (and cost) of the steel plates and instrumentation. The beam is centered halfway between the central coil hole and the left vertical edge of the plane. The criterion for the shape of the steel was to provide an adequate magnetic field in the beam region (left side) with as little steel as possible for the magnetic flux return path. Because of the near detector's squashed-octagon geometry and the offset coil, the near detector coil must carry 40,000 A-turns to achieve the specified 1.4 T magnetic field in the beam region.

The near-detector return coil is routed along the lower $45^{\circ}$ face of the uninstrumented flux-return side of the steel plane, as shown above. In the near detector there are no photodetectors or electronics on the flux return side of the steel plates so the relatively high magnetic fringe fields on that side do not affect detector operation.

A collar surrounds the magnet coil hole in each plane. This restraint serves two functions. It sets the spacing in the center of the planes to give the correct gap between planes, and it provides a smooth bore through which the magnet coil can be inserted. The coil hole in each steel plate is cut slightly larger than required for the collar. The collar can then be aligned laterally to take up the tolerance differences (e.g. imperfections in the plane assembly or misalignment of support rails). The coil hole in the center of the collar is a $26 \mathrm{~cm}$ by $26 \mathrm{~cm}$ square.

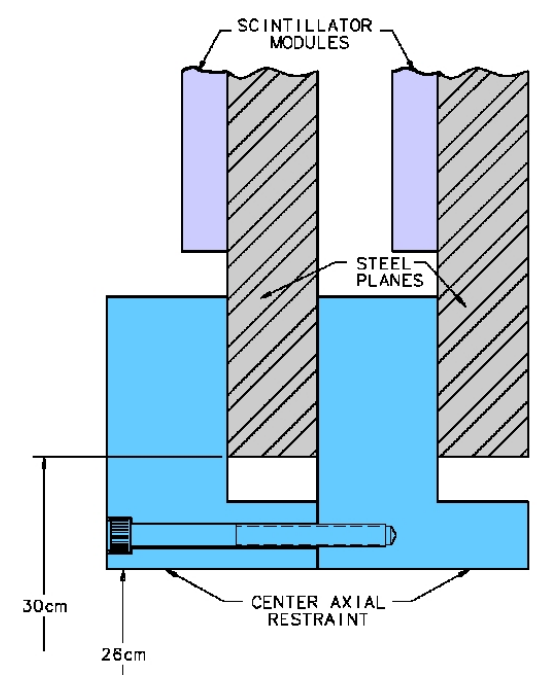

Figure 2: The section view of a steel plane coil collar showing how adjacent collars bolt together.

\section{Performance Criteria}

- Provide current of $40 \mathrm{kA}$-turns in a small package

- Minimize weight of components conductor for ease of handling

- Minimize final installation labor 
- Chose a design that fits through the NuMI drop shaft to the MINOS near detector hall

- Do not increase the temperature of the scintillation counters by more than $5^{\circ} \mathrm{C}$

- Produce a design that offers the possibility of service after coil installation

\section{Design and Properties of the Coils}

The near detector coil (which is about $18 \mathrm{~m}$ long) must carry a 40,000 A-turn current and requires substantial cooling. A low-conductivity water (LCW) closed loop provides the coil-cooling water. The LCW system for the near detector hall is provided as part of the NuMI project facility [2].

The coil consists of two approximately $18 \mathrm{~m}$ long legs (bore and return) and two approximately $4 \mathrm{~m}$ long sections to connect the bore and return legs (upstream and downstream).

We considered both copper and aluminum conductors. Both materials were examined for heat generation, power consumption, weight, ease of manufacture, and cost. Aluminum was selected to reduce the weight of the assembly. The aluminum conductor is made through the same conforming technique that was used for the $\mathrm{KTeV} \mathrm{M} \mu$-Sweep II magnet and the $\mathrm{KTeV}$ analysis magnet. In fact the same vendor, Alconix, has being considered for MINOS. The specifications and QA procedures for the conductor have been documented in the Fermilab Report NuMI-L-411 [3]. A spool containing $600 \mathrm{ft}$ of conductor was delivered earlier this year.

Each conductor is formed 1.1in $\times 1$.5in rectangular cross section aluminum with a 0.65 in diameter channel through its center. The conductor is cooled by flowing LCW through the center channel. The cross section of the one of these conductors is shown in Figure 3. The coil has 48 turns where the conductors are arranged in a 6 by 8 rectangular pattern as shown in Figure 3.

The electrical connections are made using mechanical lap joints. An example of one of these joints is shown in Figure 4. Groups of 6 conductors are formed into planks. The current flows in parallel through the conductors within a plank. The lap joints require less installation labor than designs requiring in situ welding. Another advantage of using mechanical electrical connections it that there is the potential to disassemble the coil for repair or replacement in case of failure.

Input and output water manifolds will be located at all four corners of the device. Water passes through one plank before returning to the chiller, giving $8 \times 4$ separate water circuits. The water flows in parallel within a plank. Flow calculations show that the flow rates in the different conductors within a plank are fairly well balanced [6].

There is only one electrical circuit. Each near-detector conductor carries $833 \mathrm{~A}$ and the coil is powered by a 5000A supply. 


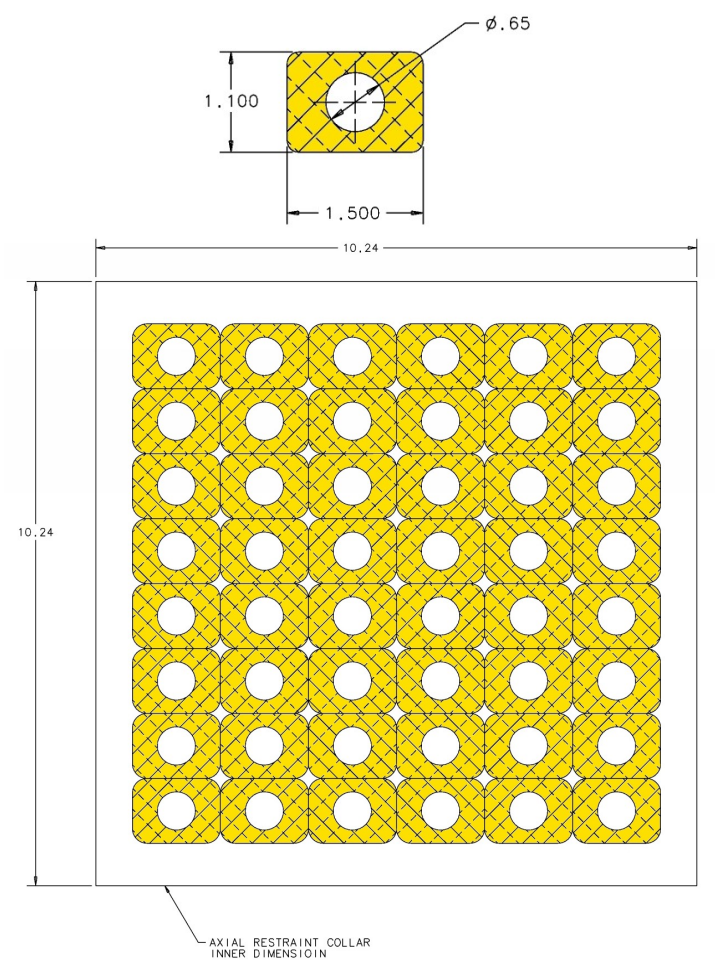

Figure 3: A cross section of one of the 48 conductors in the near detector coil (top) and a cross section of the coil (bottom). (All dimensions are in inches.)
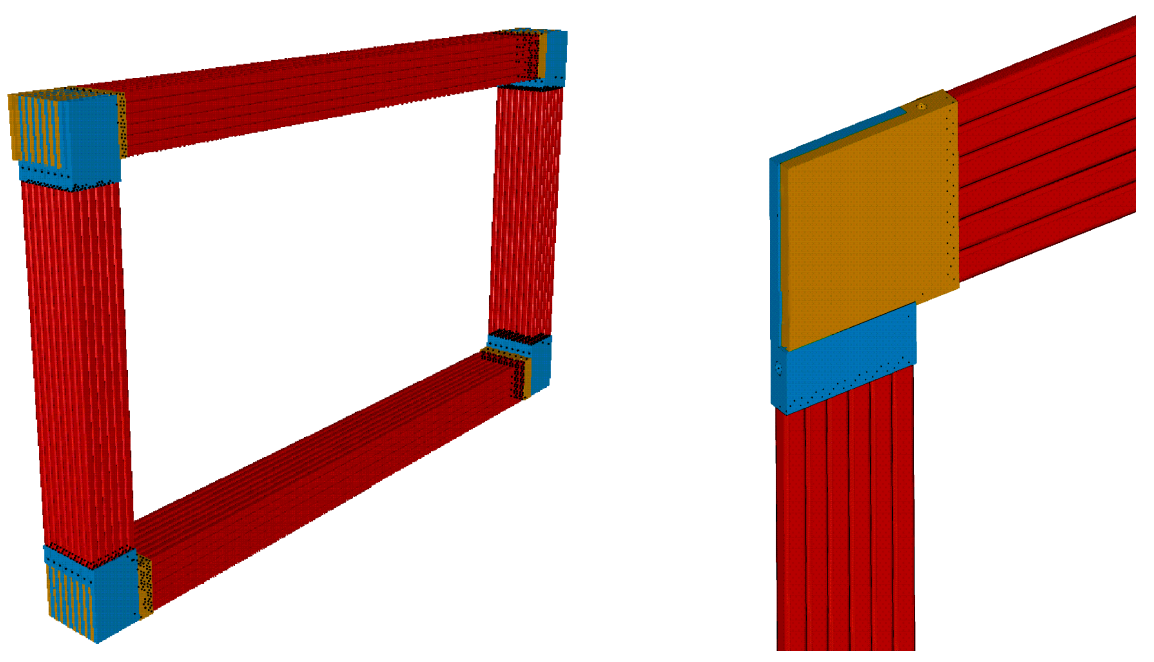

Figure 4: Sketch of the full coil assembly (left) and a sketch of one lap joint (right). The blue and gold pieces are the lap joint parts.

Thermal analyses of the planks and the lap joints have been performed [6]. Calculations show that if the total flow rate in the entire coil is 22 gallons per minute (gpm) that the temperature of the outer surface of a conductor changes by $9.4^{\circ} \mathrm{C}$ along the length of an $18 \mathrm{~m}$ plank. The pressure differential is small. With this small rate the flow was laminar. For standard calculations one assumes the flow is turbulent and that the heat is evenly mixed into the entire water volume. A 100gpm rate would to provide such a flow for the entire coil. At this flow rate the conductor's 
temperature changes by only $2.4^{\circ} \mathrm{C}$. The cooling system will be designed for a 50psi drop in the coil.

Calculations show that if you assume the resistance of the lap joint is $1000 \times$ the resistance of the bulk material, the maximum temperature increase in the lap joint is much less than $1^{\circ} \mathrm{C}$. If the resistance is $1,000,000 \times$ the resistance of the bulk material the increase is $2.8^{\circ} \mathrm{C}$.

Note that all listed temperature changes are relative to the cooling water temperature and not the ambient temperature. The LCW in the planks must be cooled by a chiller to meet the operatingtemperature specifications ( $<5^{\circ} \mathrm{C}$ change). The power bus for the coil, though, does not require the chilled LCW. The anticipated power load from the coil is $47 \mathrm{~kW}$.

\section{Near Detector Coil Manufacture and Installation}

The pre-tested aluminum conductor is delivered directly to the fabricator on spools where it is uncoiled, straightened, and cut to length. The ends of the conductor are prepared for welding in two ways: the hole is countersunk for a stainless-steel ferrel and the edges are beveled to allow for full penetration welds. The 8 conductors in a plank are welded together. The details of these preparations are summarized in the coil components and assembly drawings [5]. Ferrels are inserted into the lap-joint pieces and mated with the conductors and then welded. The planks are wrapped in two layers of B-stage epoxy and painted with epoxy paint. The finished planks are then pressure and flow tested prior to delivery to Fermilab.

After delivery the completed components are lowered into the MINOS near detector hall though a drop shaft using a special lifting fixture The drop-shaft into the NuMI near hall tunnel has been designed to allow a full 60 -foot long coil segment to be lowered into the cavern. This rigging process will be much easier before the roof of the building has been installed. Therefore, its parts are to be lowered before the hall is outfitted (spring 2001) and stored until all detector planes have been installed (summer 2002). After they are lowered, the planks are placed in wheeled storage boxes for protection.

The coil is installed after the 280 planes of the near detector have been mounted. The planks are rigged into position using a special "spreader bar" lifting fixture to support the free end of the plank as it enters the bore. Note that the center segment of the coil assembly (8 full-length planks) weighs about $500 \mathrm{~kg}$. The planks are flexible, and could be easily damaged if overstressed. After a new plank is put in place, the lap-joint connection is completed. Before the lap connections are made the surfaces will be chemically cleaned and a surface coating will be applied. There is a relatively short time (minutes) between the preparation of the surface and the mating of the splice.

Before the installation can proceed, brackets are mounted on the steel support structure to support the return coil. The coil's components include 16 long planks and 16 short planks. Installation of the first 4 planks is somewhat different than the remainder of the planks. Rollers on the lifting fixture enable the planks to slide smoothly into the bore of the detector, where it rests on insulation in the central collar tube. The long return plank is then rigged into position on the support brackets under the detector. A short plank is rigging into position on the downstream end of the detector and connected to the first two planks. Each connection will be checked for electrical integrity. The water connections to each end of the planks will also be made. A second short plank is installed on 
the upstream side of the detector and is only connected to the return plank. This completes the first circuit of planks. After this point, the installed coil sections are self-supporting. The planes will then be checked for water integrity.

The remaining planks are installed in a spiral pattern. First a bore plank is installed, then a downstream plank, then a return plank, and finally an upstream plane. As each plank is installed it is electrically connected to the previous plank. Final installation of the coil includes making connections to the power supply and tests of the entire LCW system.

\section{Coil cooling systems}

The coil cooling system for the near detector will connect to a LCW cooling system provided by the NuMI project facility. The coil cooling system will run at about $100 \mathrm{gpm}$ and must carry off the $80 \mathrm{~kW}$ of heat generated by the coil power supply, coil, and power bus. Note that only the coil itself is connected to the chiller. The supply and bus are cooled using non-chilled water.

\section{Power System}

The near detector coil a PEI 150-5 running at 5KA to deliver 5000A or 833A per conductor. The supplies are equipped with input and output filters to reduce electronic noise pickup. The power supply has remote readout and remote control capabilities. The supply, cooling system, and interlocks will be provided by the FNAL Beams Division. The supply's control and monitoring systems will be interfaced to the MINOS slow control systems.

Each coil power supply will have a current regulator and precision readout to continuously monitor its current. In addition a resistance bridge will be installed to monitor for small changes in the resistance of the coil. Thermal interlocks will also be provided in the event of excessive heating.

The power supply is also water-cooled and needs 5gpm and a maximum pressure drop of 100psi with a differential pressure of 60 PSI.

\section{Magnet Coil Installation Schedule}

As mentioned above the near detector coil components will be delivered to the near-detector cavern prior to completion of the downstream service building. The delivery date for the long sections of the coil (bore and return planks) is spring 2001 and installation will proceed in summer 2002.

\section{Magnet Coil Prototyping Plan}

To study the performance of the coil and cooling system and to optimize the installation procedure we will produce a full-current coil for the Near Detector Four-Plane Prototype (ND4PP). The ND4PP consists of 4 near-detector planes and will be built in the FY00 in the New Muon Lab at Fermilab. We will purchase the planks to connect the bore and return sections of the coil. The current stock of conductor is about half of the required conductor for these legs. Since the bore of the ND4PP is only about $1 \mathrm{ft}$ long, the bore and return planks will be non-cooled machined parts. The purchase contract could also include options for the full-length bore and return planks legs of the final coil. This coil would also allow us to perform full-field magnetics tests with the ND4PP. 


\section{References}

[1] The MINOS Collaboration, "The MINOS Detectors Technical Design Report," October 1998, Fermilab report NuMI-L-263.

[2] The Fermilab NuMI Group, “NuMI Facility Technical Design Report," September 1998, Fermilab report NuMI-346.

[3] J. Kilmer, R.W. Fast, R. Currier, and R. Stanek, " Specifications for Aluminum Conductor," September 1998, Fermilab report NuMI-L-411.

[4] The Fermilab NuMI Group, "'MINOS Cost and Schedule Plan," September 1998, Fermilab report NuMI-L-342.

[5] J. Kilmer and N. Bednar, "MINOS Near Detector Coil Plank and Assembly Drawings," August 1998, Fermilab Drawings.

[6] Z. Tang and A. Lee, "MINOS Near Detector Thermal and Hydrodynamic Modeling and Calculations," 1999, unpublished. 